\title{
INVESTIGATION WITH MODAL ANALYSIS OF EFFECTS OF HIGH PV PENETRATION ON POWER SYSTEM VOLTAGE STABILITY
}

\author{
Burak YILDIRIM * \\ ${ }^{*}$ Voc. and Tech. High School, Bingol University, Bingol, Turkey \\ ${ }^{*}$ Corresponding author; byildirim@bingol.edu.tr
}

This paper shows the effects of high PV integration on the power system voltage stability. PV power plant was applied to the IEEE 30 bus test system. Modal analysis method is used to show the effect of PV integration on power system voltage stability. The power rate of synchronous generator in the IEEE 30 bus system is increased to show the power system stability effect of high $P V$ penetration and then the $P V$ generation with the same power rate is connected appropriate bus in power system. The modal analyses of systems are done without any PV generation and with collective and scattered PV generation at various rates. In $P V$ penetration, two scenarios, namely collective and scattered are examined. The results obtained showed that the increased penetration of $P V$ system affects the power system voltage stability positively.

Key words: Modal Analysis, Photovoltaic (PV) generation, Voltage Stability, PSAT.

\section{Introduction}

The worldwide use of traditional energy sources is causing global warming, which affects our world in a negative way. Global warming is realized on the burning of fossil fuels that cause harmful gases [1]. Because of increasing environmental concerns, such as the lack of traditional fossil fuels and carbon emissions reduction, energy production from renewable energy sources is increasing considerably. PV production, which is one of the renewable energy sources, is attracting a lot of attention [2]. The total capacity of PV production plants is around $228 \mathrm{GW}$ in worldwide by the end of 2015. In China, PV production plants with a value of $15.15 \mathrm{GW}$ were established in 2015 and in total of installed value of the PV production plant reached 43.5 GW [3].

Solar Energy is the most important renewable energy source, which is still untapped in Turkey, but with a potential of minimum $500 \mathrm{GW}$. At the end of 2016, the cumulative installed PV power in Turkey reached about $832,5 \mathrm{MW}$ and increased very rapidly with a $235 \%$ growth compared to the previous year's data, 248,8 MW [4]. As in the case of turkey, the production of PV will continue to increase rapidly in the world.

The effects of PV systems on power systems have been examined in some publications. Analyzes in studies conducted at the transmission level in the literature are usually carried out on test power systems. As an example, in [5], [6], a transient stability analysis of a 9-bus power system with a 
PV production system and a oscillatory stability analysis of a 14-bus power system with a PV production system were examined. The effect of solar energy units on real distribution systems have been analyzed in the literature [7], [8]. The effect of PV penetration on the Ontario power system has been investigated in terms of the studies on large-scale PV penetration [9]. In [10], the impact of photovoltaic (PV) power plants on the power system of northern Chile is analyzed with help of timedomain simulations. Finally the impact of PV on transient and small-disturbance stability of the western interconnection in North America is analyzed [11].

The present paper investigates the voltage stability of the IEEE 30 bus test system under various PV penetration levels. A comprehensive modal analysis of the system is carried out to identify the voltage critical value of the system. The results are compared to assess the stability of the system with different PV penetration levels. To perform the studies presented in this work, Power System Analysis Toolbox (PSAT) [12] has been utilized.

This paper is generally arranged as follows. Section 2 provides brief information on the concept of voltage stability. Section 3 contains information on the modal analysis method in which the analysis work is carried out. Section 4 provides information on the PV model. The results of the numerical analysis obtained in the study are given in Section V while the last Section of the paper is devoted to the main conclusions and contributions of this paper.

\section{Voltage Stability}

Voltage stability can be expressed as the ability of the power system to maintain the voltage level within acceptable limits for all buses under normal operating conditions and after encountering a disturbance. The voltage stability is classified as small disturbance voltage stability and large disturbance voltage stability according to the size of the disturbance. Also, the time of interest for voltage stability problems can range from a few seconds to tens of minutes. For this reason, voltage stability may be a short or long-term phenomenon. It is usually more effective to classify and analyze the voltage stability as short-term voltage stability and long-term voltage stability based on the duration of the phenomenon [13].

Voltage stability can be examined using extended transient stability simulations because it is a dynamic phenomenon. Along with that, such simulations do not easily provide degree of stability or sensitivity information. As voltage stability analysis usually requires a wide range of system conditions and a large number of possible scenarios, the approaches based steady state analysis in such applications are more appropriate and can provide more information about the voltage / reactive power problem [14].

\section{Modal Analysis}

Modal analysis is a method that can be used to characterize the dynamic behavior of the power system at a operating point [15]. Modal analysis is based on the evaluation jacobian matrix of power flow equations. 
The voltage characteristics of a power system can be analyzed around an operating point by linearizing the power flow equations and analyzing the resulting sensitivity matrices. Modal analysis is the calculation of a certain number of the eigenvalues and associated eigenvectors of a reduced jacobian matrix. This matrix retains the Q-V relationships in the gird and includes the appropriate characteristics of loads, generators, and reactive power compensating devices. The magnitude of the eigenvalues associated with a mode of a voltage/reactive power change provides a relative measure of proximity to voltage instability. The eigenvalues of the matrix also indicate the different modes in which the voltage instability may occur in the system. The eigenvectors, on the other hand, provide information related to the mechanism of loss of voltage stability and information about the power grid elements and generators involved in each mode and to define the different mode format.

For the modal (or QV) analysis, 3 matrices can be used:

1. $J_{L F}$ : This matrix is derived from the static equations of power flows in transmission lines and transformers, and is generally defined as the standard power flow Jacobian matrix.

2. $J_{L F V}$ : This matrix is the complete Jacobian matrix of the power flow equations of the system.

3. $J_{L F D}$ : This matrix is computed from the complete Jacobian matrix $A_{C}$, that is defined by the linearization of the differential algebraic equation (DAE) system equations.

$$
J_{L F D}=J_{L F V}-G_{x} F_{x}^{-1} F_{y}
$$

and can thus be considered a dynamic power flow Jacobian matrix.

Algebraic variables are assumed to be only the bus voltage amplitudes and phases, i.e. $J_{L F V}=G_{y}$. If there are other algebraic variables, they can be removed from the jacobian matrix as follows.

$$
J_{L F V}=G_{y}{ }^{\left(m_{1}, m_{1}\right)}-G_{y}{ }^{\left(m_{1}, m_{2}\right)}\left[G_{y}{ }^{\left(m_{2}, m_{2}\right)}\right]^{-1} G_{y}{ }^{\left(m_{2}, m_{1}\right)}
$$

Where $m_{1}$ is twice the number of the buses of the system and $m_{2}=m-m_{1}$. The first $m_{1}$ rows of $G_{y}$ corresponds to the active and reactive power equation gradients. Thus $G_{y}$ is as follows:

$$
G_{y}=\left[\begin{array}{ll}
G_{y}{ }^{\left(m_{1}, m_{1}\right)} & G_{y}{ }^{\left(m_{1}, m_{2}\right)} \\
G_{y}{ }^{\left(m_{2}, m_{1}\right)} & G_{y}{ }^{\left(m_{2}, m_{2}\right)}
\end{array}\right]
$$

The $J_{L F D}$ matrix can be described in a manner similar to $J_{L F V}$ in equation (2). Once the power flow jacobian matrix is selected and computed, the eigenvalue analysis is applied on a reduced matrix as follows. Let's assume that the power flow Jacobian matrix is divided into four sub-matrices:

$$
J_{L F}=\left[\begin{array}{cc}
J_{P \theta} & J_{P V} \\
J_{Q \theta} & J_{Q V}
\end{array}\right]
$$

In case of the standard Jacobian matrix $J_{L F}$, this has also a physical meaning, since it can be obtained by the linearization of the power flow equations with constant power injections: 


$$
\left[\begin{array}{l}
\Delta P \\
\Delta Q
\end{array}\right]=\left[\begin{array}{ll}
J_{P \theta} & J_{P V} \\
J_{Q \theta} & J_{Q V}
\end{array}\right]\left[\begin{array}{l}
\Delta \theta \\
\Delta V
\end{array}\right]
$$

The reduced matrix is then defined as:

$$
J_{L F r}=J_{Q V}-J_{Q \theta} J_{P \theta}{ }^{-1} J_{P V}
$$

This can be used for QV sensitivity analysis.

$$
\Delta Q=J_{L F r} \Delta V
$$

Here, the stability analysis is performed using the $J_{L F r}$ matrix. If the Jacobian eigenvalues are all positive, the system is voltage stable for modal analysis. If one of the eigenvalues of the $J_{L F r}$ matrix is zero, the system reaches the voltage stability critical point. If any eigenvalue of the $J_{L F r}$ matrix is negative, the system exceeds the voltage stability critical point. Since the problem of voltage instability is not linear, the magnitudes of eigenvalues do not provide a complete measure of proximity to instability and only provide a relative relationship. The Modal analysis applications is to help in determining how much extra load or power transfer level should be added, how stable the system is and, when the system reaches voltage stability critical point, to describe the mechanism of instability by identifying elements which participate in each mode and to determine the voltage stability critical areas [12], [14], [16], [17].

\section{PV Model}

When looking at the typical structure of a grid-connected PV generator, its main subsystems are PV array, DC / AC and DC / DC converters and controllers associated with them. A DC / DC converter performs the Maximum Power Point Tracking (MPPT) requirement in PV. This converter also regulates a desired value voltage at the output of the array. PV power plant generally consists of 2 parts. In the first part solar energy is converted to electricity. In the second part, DC / AC conversion process is performed for the obtained electric energy. The voltage and/or power at the connection point is controlled by the help of the converter. The PV model is the network side because the network monitors the PV system. The PV model uses d-q display to model DC / AC power conversion. The PI controller is used to generate the reference reactive power value, which is shown in Figure 1. At the same time, the following equation 8 is used to calculate the reference currents $i_{d}$ and $i_{q}$. More detailed information on the model can be found in [18], [19].

$$
\left[\begin{array}{l}
i_{d} \\
i_{q}
\end{array}\right]=\left[\begin{array}{cc}
V_{d} & V_{q} \\
V_{q} & -V_{d}
\end{array}\right]^{-1}\left[\begin{array}{l}
P \\
Q
\end{array}\right]
$$




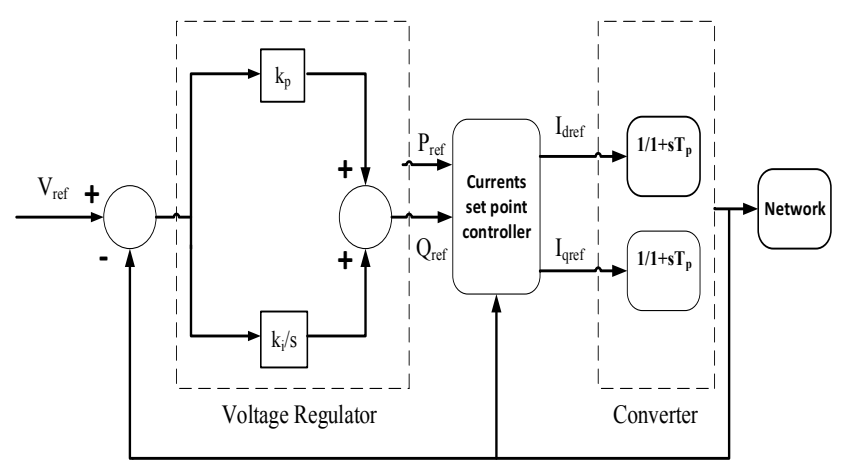

Figure 1. Photovoltaic model

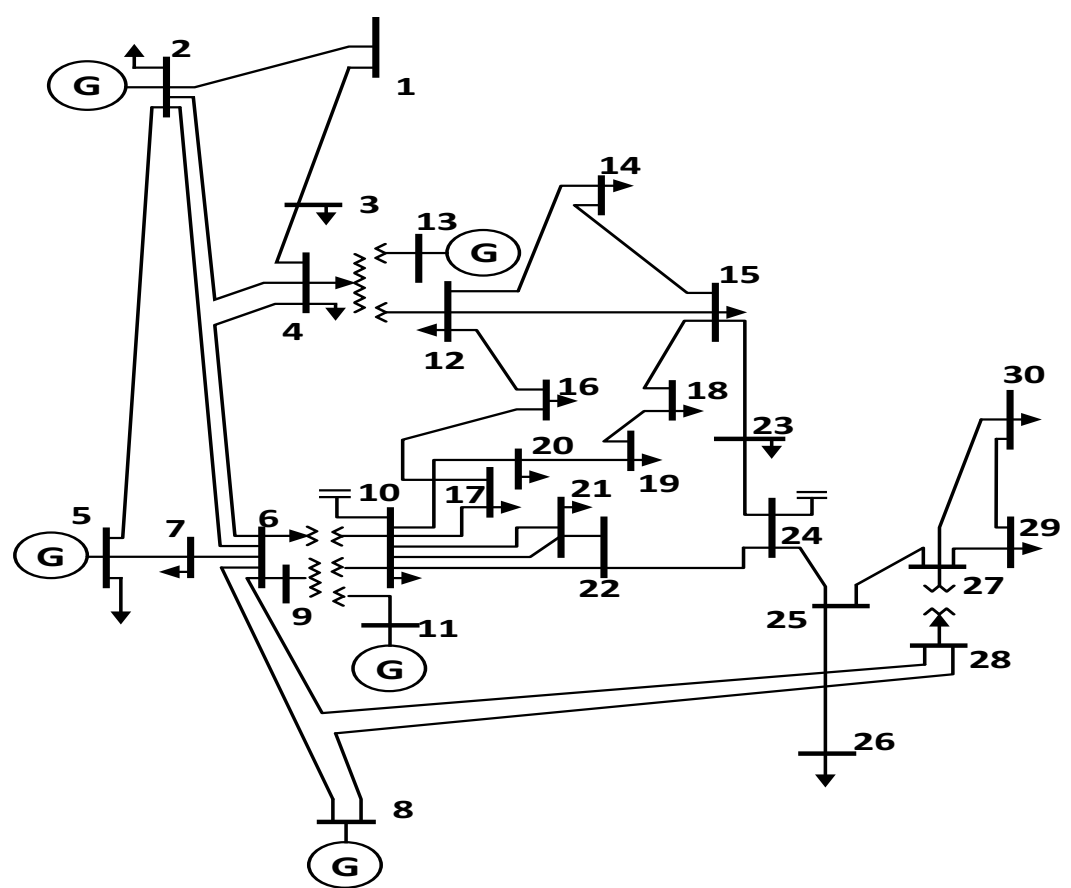

Figure 2. IEEE 30 bus test system

\section{Simulations Results}

Modal analysis has been carried out in this section to demonstrate the effects of the PV on the power system voltage stability. Figure 2 shows the IEEE 30 [20] buses test system in which the modal analysis processes are performed. The locations on PV power plants are shown in Tables I. The results of the conducted analysis are shown in Tables II, III and IV. In the base case, the power rating of the generator in bus 2 is increased 100 MVA. In this way, the total power of the production units located in the power system for the cases in which the PV integration is performed and for the basic case is set to the same value. Table II shows the results of modal analysis for the non-PV power plants. While Table III shows the results of the modal analysis for the collective PV situation, Table IV shows the results of the modal analysis for the Scattered PV situation. In the IEEE 30 buses power system, there are generators (Bus 1, 2, 5, 8, 11 and 13) in six buses and the eigenvalues of these buses are very high because the voltage is constant. Since the eigenvalues of generator buses are very high, the 6 
eigenvalues associated with these buses are not shown in Table II. Likewise, for the collective PV situation, since the PV power plant is placed on the bus 29 , value of eigenvalue 24 associated with this bus has a very high value in Table III. The same situation for the eigenvalues 22, 23 and 24 in Table IV are valid.

Table 1. Modal Analysis Results of 30 Bus Basic Power Systems

\begin{tabular}{lcc} 
Eigenvalues & Bus Location & PV Penetration \\
\hline Collective PV & Bus 29 & 100 MVA \\
Scattered PV & Bus 19 & 30 MVA \\
Scattered PV & Bus 26 & 35 MVA \\
Scattered PV & Bus 27 & 35 MVA \\
\hline
\end{tabular}

The magnitude of the eigenvalues shows that is appropriate in terms of voltage stability. As can be seen from the results of Table II, the most critical bus in terms of voltage stability is bus 30 which has the smallest eigenvalue. The value of all eigenvalues were found to be greater than zero in base power system this system is stability in terms of voltage stability. This means that all the buses have $\partial v / \partial q>0$ and as a result base power system are stable system. As Table II shows, the eigenvalue 17 is the eigenvalue which is closest to the voltage instability. The eigenvalue 17 was found to be 0.51279 in Table II. Through the sensitivity analysis, the participation rates of the buses contributing to this eigenvalue were determined. The bus that has the most effect on this eigenvalue is bus 30 with a participation rate of $22 \%$. For this reason, the closest bus to the voltage instability is the bus 30 .

The results of Table III show that the bus 26 is the most critical bus in terms of voltage stability since it has the smallest eigenvalue. In the case of collective PV integration, the power system is stable in terms of voltage stability since all eigenvalues are greater than zero. As Table III shows, the eigenvalue 9 is closest to eigenvalue with the 0.8253 value, and with the help of sensitivity analysis, the largest participation to this eigenvalue have provided 26 bus with $18 \%$ participation rates. Since the PV plant is installed on the bus 29 for the collective PV situation, the most critical bus has changed according to the basic situation and the bus 26 has been. In addition, the value of the most critical eigenvalue in terms of voltage stability has been increased from 0.51279 to 0.8253 , and the distance to the instability of power system was removed. 
Table 2. Modal Analysis Results of 30 Bus Basic Power System

\begin{tabular}{|c|c|c|c|c|c|c|c|c|c|c|c|c|c|c|c|c|c|c|c|c|}
\hline \multirow[b]{2}{*}{ Eigenvalues } & \multirow{2}{*}{$\begin{array}{l}\text { Mostly } \\
\text { associat } \\
\text { ed bus }\end{array}$} & \multirow{2}{*}{$\begin{array}{c}\text { Real and } \\
\text { Imaginary } \\
\text { parts }\end{array}$} & \multicolumn{18}{|c|}{ Participation Factors } \\
\hline & & & $\begin{array}{c}\text { Bus } \\
\mathbf{3}\end{array}$ & $\begin{array}{c}\text { Bus } \\
4\end{array}$ & $\begin{array}{c}\text { Bus } \\
6\end{array}$ & $\begin{array}{c}\text { Bus } \\
7\end{array}$ & $\begin{array}{c}\text { Bus } \\
9\end{array}$ & $\begin{array}{c}\text { Bus } \\
10\end{array}$ & $\begin{array}{c}\text { Bus } \\
12\end{array}$ & $\begin{array}{c}\text { Bus } \\
14\end{array}$ & $\begin{array}{c}\text { Bus } \\
15\end{array}$ & $\begin{array}{c}\text { Bus } \\
18\end{array}$ & $\begin{array}{c}\text { Bus } \\
19\end{array}$ & $\begin{array}{c}\text { Bus } \\
21\end{array}$ & $\begin{array}{c}\text { Bus } \\
24\end{array}$ & $\begin{array}{c}\text { Bus } \\
25\end{array}$ & $\begin{array}{c}\text { Bus } \\
26\end{array}$ & $\begin{array}{c}\text { Bus } \\
28\end{array}$ & $\begin{array}{c}\text { Bus } \\
29\end{array}$ & $\begin{array}{c}\text { Bus } \\
\mathbf{3 0}\end{array}$ \\
\hline Eig Jlfr \# 1 & Bus 06 & $110.526+0$ & 0.03 & 0.25 & 0.67 & 0.01 & 0 & 0 & 0 & 0 & 0 & 0 & 0 & 0 & 0 & 0 & 0 & 0.02 & 0 & 0 \\
\hline Eig Jlfr \# 2 & Bus 21 & $99.7576+0$ & 0 & 0 & 0 & 0 & 0 & 0.01 & 0 & 0 & 0 & 0 & 0 & 0.51 & 0 & 0 & 0 & 0 & 0 & 0 \\
\hline Eig Jlfr \# 3 & Bus 4 & $66.2165+0$ & 0.28 & 0.43 & 0.21 & 0.01 & 0 & 0 & 0.01 & 0 & 0 & 0 & 0 & 0 & 0 & 0 & 0 & 0.03 & 0 & 0 \\
\hline Eig Jlfr \# 4 & Bus 10 & $59.2487+0$ & 0 & 0 & 0 & 0 & 0.04 & 0.77 & 0 & 0 & 0 & 0 & 0 & 0.01 & 0 & 0 & 0 & 0 & 0 & 0 \\
\hline Eig Jlfr \# 5 & Bus 12 & $44.5048+0$ & 0.01 & 0 & 0 & 0 & 0 & 0 & 0.7 & 0 & 0.17 & 0 & 0 & 0 & 0 & 0 & 0 & 0 & 0 & 0 \\
\hline Eig Jlfr \# 6 & Bus 19 & $37.2957+0$ & 0 & 0 & 0 & 0 & 0 & 0 & 0 & 0 & 0 & 0.05 & 0.55 & 0 & 0 & 0 & 0 & 0 & 0 & 0 \\
\hline Eig Jlfr \# 7 & Bus 15 & $25.2468+0$ & 0 & 0 & 0 & 0 & 0 & 0 & 0.05 & 0.06 & 0.55 & 0.05 & 0 & 0 & 0.01 & 0 & 0 & 0 & 0 & 0 \\
\hline Eig Jlfr \# 8 & Bus 28 & $23.1249+0$ & 0.02 & 0 & 0 & 0.22 & 0 & 0 & 0 & 0 & 0 & 0 & 0 & 0 & 0 & 0 & 0 & 0.37 & 0 & 0 \\
\hline Eig Jlfr \# 9 & Bus 9 & $19.6491+0$ & 0 & 0 & 0 & 0.14 & 0.49 & 0 & 0.02 & 0.01 & 0.01 & 0 & 0 & 0 & 0.01 & 0 & 0 & 0 & 0 & 0 \\
\hline Eig Jlfr \# 10 & Bus 7 & $19.1542+0$ & 0.14 & 0.03 & 0 & 0.33 & 0 & 0 & 0.01 & 0.01 & 0.01 & 0.01 & 0 & 0.02 & 0.04 & 0.02 & 0 & 0.02 & 0 & 0 \\
\hline Eig Jlfr \# 11 & Bus 24 & $18.0978+0$ & 0.11 & 0.03 & 0 & 0.11 & 0.11 & 0 & 0.01 & 0.01 & 0.01 & 0.01 & 0 & 0.07 & 0.2 & 0.06 & 0 & 0.05 & 0 & 0 \\
\hline Eig Jlfr \# 12 & Bus 25 & $16.5696+0$ & 0 & 0 & 0 & 0 & 0.18 & 0.01 & 0.01 & 0 & 0 & 0 & 0 & 0.01 & 0.06 & 0.27 & 0 & 0.01 & 0 & 0 \\
\hline Eig Jlfr \# 13 & Bus 24 & $13.939+0$ & 0.06 & 0.02 & 0 & 0 & 0.03 & 0.01 & 0 & 0 & 0.01 & 0.04 & 0.01 & 0.01 & 0.28 & 0.04 & 0 & 0 & 0.01 & 0 \\
\hline Eig Jlfr \# 14 & Bus 18 & $13.5044+0$ & 0 & 0 & 0 & 0 & 0 & 0 & 0 & 0 & 0.02 & 0.49 & 0.03 & 0.01 & 0.02 & 0 & 0 & 0 & 0 & 0 \\
\hline Eig Jlfr \# 15 & Bus 3 & $11.1728+0$ & 0.29 & 0.18 & 0.07 & 0.12 & 0.03 & 0 & 0 & 0 & 0 & 0 & 0 & 0 & 0 & 0.03 & 0 & 0.13 & 0 & 0 \\
\hline Eig Jlfr \# 16 & Bus 14 & $9.8045+0$ & 0.01 & 0 & 0 & 0 & 0 & 0 & 0.01 & 0.34 & 0 & 0 & 0 & 0.06 & 0.01 & 0 & 0 & 0 & 0 & 0 \\
\hline Eig Jlfr \# 17 & Bus 30 & $0.51279+0$ & 0 & 0 & 0 & 0 & 0 & 0 & 0 & 0 & 0 & 0.01 & 0.01 & 0.01 & 0.03 & 0.1 & 0.17 & 0 & 0.20 & 0.22 \\
\hline Eig Jlfr \# 18 & Bus 19 & $1.0632+0$ & 0 & 0 & 0 & 0 & 0.01 & 0.04 & 0.02 & 0.04 & 0.05 & 0.11 & 0.12 & 0.03 & 0.03 & 0 & 0 & 0 & 0.08 & 0.10 \\
\hline Eig Jlfr \# 19 & Bus 26 & $1.7527+0$ & 0 & 0 & 0 & 0 & 0 & 0 & 0 & 0 & 0 & 0.01 & 0.01 & 0 & 0 & 0.05 & 0.62 & 0 & 0.10 & 0.14 \\
\hline Eig Jlfr \# 20 & Bus 25 & $7.5335+0$ & 0 & 0 & 0 & 0 & 0 & 0 & 0 & 0.03 & 0 & 0 & 0 & 0 & 0 & 0.25 & 0.06 & 0.01 & 0.15 & 0 \\
\hline Eig Jlfr \# 21 & Bus 19 & $3.6612+0$ & 0 & 0 & 0 & 0 & 0 & 0.02 & 0 & 0 & 0 & 0.15 & 0.21 & 0.07 & 0.1 & 0 & 0.04 & 0 & 0 & 0 \\
\hline Eig Jlfr \# 22 & Bus 14 & $4.1381+0$ & 0 & 0 & 0 & 0 & 0.02 & 0.06 & 0.05 & 0.32 & 0.12 & 0 & 0.01 & 0.09 & 0 & 0 & 0 & 0 & 0 & 0 \\
\hline Eig Jlfr \# 23 & Bus 29 & $6.1929+0$ & 0 & 0 & 0 & 0 & 0 & 0 & 0 & 0.02 & 0 & 0 & 0 & 0 & 0.03 & 0 & 0 & 0 & 0.38 & 0.35 \\
\hline Eig Jlfr \# 24 & Bus 30 & $5.7543+0$ & 0 & 0 & 0 & 0 & 0.01 & 0.01 & 0.02 & 0.06 & 0 & 0.01 & 0 & 0 & 0.09 & 0.1 & 0.06 & 0 & 0.03 & 0.15 \\
\hline
\end{tabular}

Table 3. Modal Analysis Results of 30 Bus Power System with Collective PV

\begin{tabular}{|c|c|c|c|c|c|c|c|c|c|c|c|c|c|c|c|c|c|c|c|c|}
\hline \multirow[b]{2}{*}{ Eigenvalues } & \multirow{2}{*}{$\begin{array}{c}\text { Mostly } \\
\text { associat } \\
\text { ed bus }\end{array}$} & \multirow{2}{*}{$\begin{array}{c}\text { Real and } \\
\text { Imaginary } \\
\text { parts }\end{array}$} & \multicolumn{18}{|c|}{ Participation Factors } \\
\hline & & & $\begin{array}{c}\text { Bus } \\
\mathbf{3}\end{array}$ & $\begin{array}{c}\text { Bus } \\
4\end{array}$ & $\begin{array}{c}\text { Bus } \\
6\end{array}$ & $\begin{array}{c}\text { Bus } \\
7 \\
\end{array}$ & $\begin{array}{c}\text { Bus } \\
9\end{array}$ & $\begin{array}{c}\text { Bus } \\
10\end{array}$ & $\begin{array}{c}\text { Bus } \\
12 \\
\end{array}$ & $\begin{array}{c}\text { Bus } \\
14\end{array}$ & $\begin{array}{c}\text { Bus } \\
15 \\
\end{array}$ & $\begin{array}{c}\text { Bus } \\
18\end{array}$ & $\begin{array}{c}\text { Bus } \\
19 \\
\end{array}$ & $\begin{array}{c}\text { Bus } \\
21\end{array}$ & $\begin{array}{c}\text { Bus } \\
24 \\
\end{array}$ & $\begin{array}{c}\text { Bus } \\
25 \\
\end{array}$ & $\begin{array}{c}\text { Bus } \\
26 \\
\end{array}$ & $\begin{array}{c}\text { Bus } \\
28 \\
\end{array}$ & $\begin{array}{c}\text { Bus } \\
29 \\
\end{array}$ & $\begin{array}{c}\text { Bus } \\
\mathbf{3 0}\end{array}$ \\
\hline Eig Jlfr \# 1 & Bus 6 & $110.742+0$ & 0.03 & 0.25 & 0.67 & 0.01 & 0 & 0 & 0 & 0 & 0 & 0 & 0 & 0 & 0 & 0 & 0 & 0.02 & 0 & 0 \\
\hline Eig Jlfr \# 2 & Bus 21 & $98.7654+0$ & 0 & 0 & 0 & 0 & 0 & 0.01 & 0 & 0 & 0 & 0 & 0 & 0.51 & 0 & 0 & 0 & 0 & 0 & 0 \\
\hline Eig Jlfr \# 3 & Bus 4 & $66.3638+0$ & 0.28 & 0.43 & 0.21 & 0.01 & 0 & 0 & 0.01 & 0 & 0 & 0 & 0 & 0 & 0 & 0 & 0 & 0.03 & 0 & 0 \\
\hline Eig Jlfr \# 4 & Bus 10 & $58.7807+0$ & 0 & 0 & 0 & 0 & 0.04 & 0.77 & 0 & 0 & 0 & 0 & 0 & 0.01 & 0 & 0 & 0 & 0 & 0 & 0 \\
\hline Eig Jlfr \# 5 & Bus 12 & $44.3061+0$ & 0.01 & 0 & 0 & 0 & 0 & 0 & 0.7 & 0 & 0.17 & 0 & 0 & 0 & 0 & 0 & 0 & 0 & 0 & 0 \\
\hline Eig Jlfr \# 6 & Bus 19 & $37.0038+0$ & 0 & 0 & 0 & 0 & 0 & 0 & 0 & 0 & 0 & 0.05 & 0.55 & 0 & 0 & 0 & 0 & 0 & 0 & 0 \\
\hline Eig Jlfr \# 7 & Bus 15 & $25.0718+0$ & 0 & 0 & 0 & 0 & 0 & 0 & 0.05 & 0.06 & 0.55 & 0.05 & 0 & 0 & 0.01 & 0 & 0 & 0 & 0 & 0 \\
\hline Eig Jlfr \# 8 & Bus 28 & $23.1307+0$ & 0.02 & 0 & 0 & 0.22 & 0 & 0 & 0 & 0 & 0 & 0 & 0 & 0 & 0 & 0 & 0 & 0.67 & 0 & 0 \\
\hline Eig Jlfr \# 9 & Bus 26 & $0.8253+0$ & 0 & 0 & 0 & 0 & 0.01 & 0.03 & 0.01 & 0.03 & 0.04 & 0.07 & 0.08 & 0.05 & 0.07 & 0.07 & 0.18 & 0 & 0 & 0 \\
\hline Eig Jlfr \# 10 & Bus 26 & $1.4029+0$ & 0 & 0 & 0 & 0 & 0 & 0.01 & 0 & 0.02 & 0.02 & 0.06 & 0.06 & 0.01 & 0 & 0.09 & 0.53 & 0 & 0 & 0.01 \\
\hline Eig Jlfr \# 11 & Bus 30 & $3.2333+0$ & 0 & 0 & 0 & 0 & 0 & 0 & 0 & 0 & 0 & 0 & 0 & 0 & 0 & 0 & 0.1 & 0 & 0 & 0.77 \\
\hline Eig Jlfr \# 12 & Bus 19 & $3.6453+0$ & 0 & 0 & 0 & 0 & 0 & 0.02 & 0 & 0 & 0 & 0.14 & 0.19 & 0.06 & 0.09 & 0 & 0.02 & 0 & 0 & 0.07 \\
\hline Eig Jlfr \# 14 & Bus 23 & $5.7075+0$ & 0 & 0 & 0 & 0 & 0.01 & 0.02 & 0.02 & 0.07 & 0 & 0.01 & 0.01 & 0 & 0.11 & 0.12 & 0.07 & 0 & 0 & 0.04 \\
\hline Eig Jlfr \# 15 & Bus 27 & $7.0483+0$ & 0 & 0 & 0 & 0 & 0 & 0 & 0.01 & 0.05 & 0 & 0 & 0 & 0 & 0.02 & 0.19 & 0.05 & 0.01 & 0 & 0.06 \\
\hline Eig Jlfr \# 16 & Bus 9 & $19.5702+0$ & 0 & 0 & 0 & 0.17 & 0.48 & 0 & 0.02 & 0.01 & 0.01 & 0 & 0 & 0 & 0.01 & 0 & 0 & 0.01 & 0 & 0 \\
\hline Eig Jlfr \# 17 & Bus 7 & $19.0852+0$ & 0.17 & 0.04 & 0 & 0.34 & 0 & 0 & 0.01 & 0.01 & 0.01 & 0 & 0 & 0.01 & 0.02 & 0.01 & 0 & 0.03 & 0 & 0 \\
\hline Eig Jlfr \# 18 & Bus 24 & $17.9599+0$ & 0.09 & 0.02 & 0 & 0.08 & 0.13 & 0 & 0.01 & 0.01 & 0.01 & 0.01 & 0 & 0.09 & 0.21 & 0.05 & 0 & 0.04 & 0 & 0 \\
\hline Eig Jlfr \# 19 & Bus 25 & $16.2274+0$ & 0 & 0 & 0 & 0 & 0.17 & 0.01 & 0.01 & 0 & 0 & 0 & 0 & 0.01 & 0.1 & 0.29 & 0 & 0 & 0 & 0 \\
\hline Eig Jlfr \# 20 & Bus 14 & $9.7322+0$ & 0 & 0 & 0 & 0 & 0 & 0 & 0.01 & 0.34 & 0 & 0 & 0 & 0.05 & 0.01 & 0 & 0 & 0 & 0 & 0 \\
\hline Eig Jlfr \# 21 & Bus 3 & $11.1886+0$ & 0.28 & 0.17 & 0.07 & 0.12 & 0.04 & 0 & 0 & 0 & 0 & 0 & 0 & 0 & 0 & 0.03 & 0 & 0.13 & 0 & 0 \\
\hline Eig Jlfr \# 22 & Bus 24 & $13.6422+0$ & 0.05 & 0.02 & 0 & 0 & 0.02 & 0.01 & 0 & 0 & 0.02 & 0.1 & 0.02 & 0.02 & 0.21 & 0.06 & 0 & 0 & 0 & 0 \\
\hline Eig Jlfr \# 23 & Bus 18 & $13.3741+0$ & 0.01 & 0 & 0 & 0 & 0 & 0 & 0 & 0.03 & 0.01 & 0.42 & 0.02 & 0 & 0.05 & 0.01 & 0 & 0 & 0 & 0 \\
\hline Eig Jlfr \# 24 & Bus 29 & $999+0$ & 0 & 0 & 0 & 0 & 0 & 0 & 0 & 0 & 0 & 0 & 0 & 0 & 0 & 0 & 0 & 0 & 1 & 0 \\
\hline
\end{tabular}


Table 4. Modal Analysis Results of 30 Bus Power System with Scattered PV

\begin{tabular}{|c|c|c|c|c|c|c|c|c|c|c|c|c|c|c|c|c|c|c|c|c|c|c|c|}
\hline \multirow[b]{2}{*}{ Eigenvalues } & \multirow{2}{*}{$\begin{array}{l}\text { Mostly } \\
\text { associat } \\
\text { ed bus }\end{array}$} & \multirow{2}{*}{$\begin{array}{c}\text { Real and } \\
\text { Imaginary } \\
\text { parts }\end{array}$} & \multicolumn{21}{|c|}{ Participation Factors } \\
\hline & & & $\begin{array}{c}\text { Bus } \\
\mathbf{3}\end{array}$ & $\begin{array}{c}\text { Bus } \\
4\end{array}$ & $\begin{array}{c}\text { Bus } \\
6\end{array}$ & $\begin{array}{c}\text { Bus } \\
7\end{array}$ & $\begin{array}{c}\text { Bus } \\
9\end{array}$ & $\begin{array}{c}\text { Bus } \\
10\end{array}$ & $\begin{array}{c}\text { Bus } \\
12\end{array}$ & $\begin{array}{c}\text { Bus } \\
14\end{array}$ & $\begin{array}{c}\text { Bus } \\
15\end{array}$ & $\begin{array}{c}\text { Bus } \\
18\end{array}$ & $\begin{array}{c}\text { Bus } \\
19\end{array}$ & $\begin{array}{c}\text { Bus } \\
20\end{array}$ & $\begin{array}{c}\text { Bus } \\
21\end{array}$ & $\begin{array}{c}\text { Bus } \\
23\end{array}$ & $\begin{array}{c}\text { Bus } \\
24\end{array}$ & $\begin{array}{c}\text { Bus } \\
25\end{array}$ & $\begin{array}{c}\text { Bus } \\
26\end{array}$ & $\begin{array}{c}\text { Bus } \\
27\end{array}$ & $\begin{array}{c}\text { Bus } \\
28\end{array}$ & $\begin{array}{c}\text { Bus } \\
29\end{array}$ & $\begin{array}{c}\text { Bus } \\
30\end{array}$ \\
\hline Eig JIfr \# 1 & Bus 6 & $111.111+0$ & 0.03 & 0.25 & 0.67 & 0.01 & 0 & 0 & 0 & 0 & 0 & 0 & 0 & 0 & 0 & 0 & 0 & 0 & 0 & 0 & 0.02 & 0 & 0 \\
\hline Eig Jlfr \# 2 & Bus 21 & $98.9019+0$ & 0 & 0 & 0 & 0 & 0 & 0.01 & 0 & 0 & 0 & 0 & 0 & 0 & 0.51 & 0 & 0 & 0 & 0 & 0 & 0 & 0 & 0 \\
\hline Eig Jlfr \# 3 & Bus 4 & $66.596+0$ & 0.28 & 0.43 & 0.21 & 0.01 & 0 & 0 & 0 & 0 & 0 & 0 & 0 & 0 & 0 & 0 & 0 & 0 & 0 & 0 & 0.03 & 0 & 0 \\
\hline Eig Jlfr \# 4 & Bus 10 & $58.6969+0$ & 0 & 0 & 0 & 0 & 0.04 & 0.78 & 0 & 0 & 0 & 0 & 0 & 0.01 & 0.01 & 0 & 0 & 0 & 0 & 0 & 0 & 0 & 0 \\
\hline Eig Jlfr \# 5 & Bus 12 & $44.3158+0$ & 0.01 & 0 & 0 & 0 & 0 & 0 & 0.7 & 0 & 0.17 & 0 & 0 & 0 & 0 & 0 & 0 & 0 & 0 & 0 & 0 & 0 & 0 \\
\hline Eig Jlfr \# 6 & Bus 15 & $25.2493+0$ & 0 & 0 & 0 & 0 & 0 & 0 & 0.05 & 0.06 & 0.55 & 0.07 & 0 & 0 & 0 & 0.06 & 0 & 0 & 0 & 0 & 0 & 0 & 0 \\
\hline Eig Jlfr \# 7 & Bus 28 & $22.8435+0$ & 0.02 & 0 & 0 & 0.26 & 0 & 0 & 0 & 0 & 0 & 0 & 0 & 0 & 0 & 0 & 0 & 0 & 0 & 0 & 0.68 & 0 & 0 \\
\hline Eig Jlfr \# 8 & Bus 20 & $19.7092+0$ & 0 & 0 & 0 & 0.04 & 0.05 & 0 & 0 & 0 & 0 & 0 & 0 & 0.83 & 0 & 0 & 0 & 0 & 0 & 0 & 0 & 0 & 0 \\
\hline Eig Jlfr \# 9 & Bus 9 & $19.5622+0$ & 0 & 0 & 0 & 0.1 & 0.47 & 0 & 0.03 & 0.01 & 0.01 & 0 & 0 & 0.01 & 0 & 0 & 0 & 0 & 0 & 0 & 0.01 & 0 & 0 \\
\hline Eig Jlfr \# 10 & Bus 7 & $18.929+0$ & 0.21 & 0.05 & 0 & 0.34 & 0 & 0 & 0 & 0 & 0 & 0 & 0 & 0.07 & 0.01 & 0 & 0.01 & 0 & 0 & 0 & 0.07 & 0 & 0 \\
\hline Eig Jlfr \# 11 & Bus 24 & $17.9288+0$ & 0.05 & 0.01 & 0 & 0.05 & 0.13 & 0 & 0.01 & 0.01 & 0.02 & 0.01 & 0 & 0 & 0.12 & 0.01 & 0.24 & 0.04 & 0 & 0 & 0.01 & 0 & 0 \\
\hline Eig Jlfr \# 12 & Bus 24 & $15.0643+0$ & 0.01 & 0 & 0 & 0 & 0.15 & 0.03 & 0.01 & 0 & 0 & 0 & 0 & 0.03 & 0 & 0.09 & 0.32 & 0.13 & 0 & 0 & 0 & 0 & 0 \\
\hline Eig Jlfr \# 13 & Bus 3 & $11.3279+0$ & 0.33 & 0.2 & 008 & 0.14 & 0.03 & 0 & 0 & 0 & 0 & 0 & 0 & 0 & 0 & 0 & 0 & 0 & 0 & 0 & 0.13 & 0 & 0 \\
\hline Eig Jlfr \# 14 & Bus 18 & $11.5684+0$ & 0 & 0 & 0 & 0 & 0 & 0 & 0 & 0.04 & 0.02 & 0.82 & 0 & 0 & 0 & 0.04 & 0 & 0.02 & 0 & 0 & 0 & 0 & 0 \\
\hline Eig Jlfr \# 15 & Bus 25 & $10.0535+0$ & 0 & 0 & 0 & 0 & 0 & 0 & 0.02 & 0.11 & 0 & 0 & 0 & 0 & 0.07 & 0 & 0 & 0.32 & 0 & 0 & 0 & 0 & 0 \\
\hline Eig Jlfr \# 16 & Bus 25 & $9.4014+0$ & 0 & 0 & 0 & 0 & 0 & 0 & 0 & 0.25 & 0 & 0.01 & 0 & 0 & 0 & 0.23 & 0.03 & 0.36 & 0 & 0 & 0 & 0 & 0 \\
\hline Eig Jlfr \# 17 & Bus 14 & $4.0702+0$ & 0 & 0 & 0 & 0 & 0.02 & 0.06 & 0.04 & 0.29 & 0.11 & 0.03 & 0 & 0 & 0.12 & 0.07 & 0.02 & 0 & 0 & 0 & 0 & 0 & 0 \\
\hline Eig Jlfr \# 18 & Bus 23 & $5.8001+0$ & 0 & 0 & 0 & 0 & 0.01 & 0.02 & 0.03 & 0.07 & 0 & 0 & 0 & 0 & 0 & 0.31 & 0.19 & 0.08 & 0 & 0 & 0 & 0 & 0 \\
\hline Eig Jlfr \# 19 & Bus 23 & $1.8918+0$ & 0 & 0 & 0 & 0 & 0.02 & 0.06 & 0.04 & 0.09 & 0.07 & 0.01 & 0 & 0 & 0.1 & 0.15 & 0.12 & 0.01 & 0 & 0 & 0 & 0 & 0 \\
\hline Eig Jlfr \# 20 & Bus 30 & $1.9443+0$ & 0 & 0 & 0 & 0 & 0 & 0 & 0 & 0 & 0 & 0 & 0 & 0 & 0 & 0 & 0 & 0 & 0 & 0 & 0 & 0.4 & 0.59 \\
\hline Eig Jlfr \# 21 & Bus 29 & $6.3622+0$ & 0 & 0 & 0 & 0 & 0 & 0 & 0 & 0 & 0 & 0 & 0 & 0 & 0 & 0 & 0 & 0 & 0 & 0 & 0 & 0.59 & 0.4 \\
\hline Eig Jlfr \# 22 & Bus 19 & $999+0$ & 0 & 0 & 0 & 0 & 0 & 0 & 0 & 0 & 0 & 0 & 1 & 0 & 0 & 0 & 0 & 0 & 0 & 0 & 0 & 0 & 0 \\
\hline Eig Jlfr \# 23 & Bus 26 & $999+0$ & 0 & 0 & 0 & 0 & 0 & 0 & 0 & 0 & 0 & 0 & 0 & 0 & 0 & 0 & 0 & 0 & 1 & 0 & 0 & 0 & 0 \\
\hline Eig Jlfr \# 24 & Bus 27 & $999+0$ & 0 & 0 & 0 & 0 & 0 & 0 & 0 & 0 & 0 & 0 & 0 & 0 & 0 & 0 & 0 & 0 & 0 & 1 & 0 & 0 & 0 \\
\hline
\end{tabular}

The results of Table IV show that the bus 23 is the most critical bus in terms of voltage stability since it has the smallest eigenvalue. In the case of scattered PV integration, the power system is stable in terms of voltage stability since all eigenvalues are greater than zero. As Table IV shows, the eigenvalue 19 is closest to eigenvalue with the 1.8918 value, and with the help of sensitivity analysis, the largest participation to this eigenvalue have provided 23 bus with $15 \%$ participation rates. Since the PV plants are installed on the bus 19, 26 and 27 for the scattered PV situation, the most critical bus has changed according to the basic and collective PV situation and thus the bus 23 has been. In addition, the value of the most critical eigenvalue in terms of voltage stability has been increased from 0.51279 and 0.8253 to 1.8918 , and the distance to the instability of power system was removed.

As can be seen from this result, in terms of voltage stability, PV integration has been positively influenced on the power system and provided the healing effects in the distance of the critical bus to the voltage instability. When the results of Table III and Table IV are compared, it is seen that the scattered PV integration state provides more positive results than the collective PV integration state. Because the most critical eigenvalue for Table IV is 1.8918, this value is found to be 0.8253 in Table III. 


\section{Conclusions}

The PV integration effect on the voltage stability is discussed and examined in this paper. The studies have been made for the IEEE 30 bus test system. A comprehensive modal analysis of the system has been carried out to identify the voltage stability critical value of the system. To perform the studies presented in this work, PSAT has been utilized. The results of the analysis showed that the PV integration has positively effects on voltage stability of power system by increasing voltage instability limit value for critic bus of power systems in which PV power plant are placed. From the results of the analysis it can be observed that, scattered PV penetration increases the distance of the critical bus to the voltage instability than the collective PV penetration in IEEE-30 bus test system.

\section{REFERENCES}

[1] B. Singh, S. Kumar, and C. Jain, "Damped-SOGI Based Control Algorithm For Solar PV Power Generating System," IEEE Trans. Ind. Appl., vol. 53, no. 3, pp. 1780-1788, 2017.

[2] T. Chaiyatham and I. Ngamroo, "Improvement of power system transient stability by PV farm with fuzzy gain scheduling of PID controller," IEEE Syst. J., vol. 11, no. 3, pp. 1684-1691, 2017.

[3] "The IEA PVPS Report Trends 2016 in Photovoltaic Applications.," 2016.

[4] “IEA-PVPS Annual Report 2016," 2017.

[5] M. Yagami, N. Kimura, M. Tsuchimoto, and J. Tamura, "Power System Transient Stability Analysis in the Case of High-Penetration Photovoltaics," in PowerTech, 2013.

[6] R. Shah, N. Mithulananthan, A. Sode-Yome, and K. Y. Lee, "Impact of large-scale PV penetration on power system oscillatory stability," in IEEE PES General Meeting, 2010, pp. 17.

[7] D. Santos-Martin, S. Lemon, J. D. Watson, A. R. Wood, A. J. V. Miller, and N. R. Watson, "Impact of solar photovoltaics on the low-voltage distribution network in New Zealand," IET Gener. Transm. Distrib., vol. 10, no. 1, pp. 1-9, 2016.

[8] T. K. Saha, M. I. Hossain, P. Meredith, A. Ananth, and R. Yan, "Megawatt-scale solar variability study: an experience from a $1.2 \mathrm{MWp}$ photovoltaic system in Australia over three years," IET Renew. Power Gener., vol. 10, no. 8, pp. 1229-1236, 2016.

[9] B. Tamimi, C. Cañizares, and K. Bhattacharya, "System stability impact of large-scale and distributed solar photovoltaic generation: The case of Ontario, Canada," IEEE Trans. Sustain. Energy, vol. 4, no. 3, pp. 680-688, 2013.

[10] D. Remon, C. A. Cañizares, and P. Rodriguez, "Impact of 100-MW-scale PV plants with synchronous power controllers on power system stability in northern Chile," IET Gener. Transm. Distrib., vol. 11, no. 11, pp. 2958-2964, 2017.

[11] S. Eftekharnejad, V. Vittal, G. T. Heydt, B. Keel, and J. Loehr, "Small Signal Stability Assessment of Power Systems With Increased Penetration of Photovoltaic Generation: A Case Study," Ieee Trans. Sustain. Energy, vol. 4, no. 4, pp. 960-967, 2013.

[12] F. Milano, "PSAT Power System Analysis Toolbox," p. 446, 2008.

[13] P. Kundur et al., "Definition and Classification of Power System Stability IEEE/CIGRE Joint Task Force on Stability Terms and Definitions," IEEE Trans. Power Syst., vol. 19, no. 3, pp. 1387-1401, 2004.

[14] B. Gao, G. K. Morison, and P. Kundur, "Voltage stability evaluation using modal analysis," IEEE Trans. Power Syst., vol. 7, no. 4, pp. 1529-1542, 1992.

[15] F. Dussaud, "An application of modal analysis in electric power systems to study inter-area oscillations," KTH Royal Institute of Technology, 2015.

[16] W. Xu and Y. Mansour, "Voltage stability analysis using generic dynamic load models," Power Syst. IEEE Trans., vol. 9, no. 1, pp. 479-493, 1994.

[17] G. K. Morison, B. Gao, and P. Kundur, "Voltage stability analysis using static and dynamic 
approaches,” Power Syst. IEEE Trans., vol. 8, no. 3, pp. 1159-1171, 1993.

[18] B. Tamimi, C. Cañizares, and K. Bhattacharya, "Modeling and Performance Analysis of Large Solar Photo-Voltaic Generation on Voltage Stability and Inter-area Oscillations," Power Energy Soc. Gen. Meet. 2011 IEEE, pp. 1-6, 2011.

[19] M. A. Jagtap and S. L. Shaikh, "The Effect of High PV Penetration on The Small Signal Stability of the Power System Using PSAT," Proceedings of ITResearch International Conference,. Kolhapur, India, pp. 33-37, 2015.

[20] "Power Systems Test Case Archive." [Online]. Available: https://www2.ee.washington.edu/research/pstca/pf30/pg_tca30bus.htm. [Accessed: 14-Nov2017]. 